\begin{tabular}{|c|c|c|}
\hline Epoche & I9II & M.Z.Gr. \\
\hline 2466 & Sept. 9 & 8.4 \\
\hline 67 & $\gg \quad$ I 3 & 2.8 \\
\hline 68 & I 6 & $2 \mathrm{I} .2$ \\
\hline 69 & 20 & 15.5 \\
\hline 2470 & 24 & 9.9 \\
\hline $7 \mathrm{I}$ & $》 \quad 28$ & $4 \cdot 3$ \\
\hline 72 & Okt. I & 22.7 \\
\hline 73 & 5 & 17.0 \\
\hline
\end{tabular}

\begin{tabular}{|c|c|c|}
\hline Epoche & I9I I & M.Z.Gr. \\
\hline 2474 & Okt. $\quad 9$ & I I ${ }^{\mathrm{h}} \cdot 4$ \\
\hline 75 & I 3 & 5.8 \\
\hline $7^{6}$ & $» \quad x 7$ & 0.2 \\
\hline 77 & $\gg \quad 20$ & I 8.6 \\
\hline 78 & $》 \quad 24$ & I 2.9 \\
\hline 79 & $2 \quad 28$ & $7 \cdot 3$ \\
\hline 2480 & Nov. I & 1.7 \\
\hline $8 \mathrm{I}$ & $》$ & 20.1 \\
\hline
\end{tabular}

\begin{tabular}{|c|c|c|}
\hline Epoche & I9 I I & M.Z.Gr. \\
\hline 2482 & Nov. 8 & $I 4^{h} \cdot 4$ \\
\hline 83 & I 2 & 8.8 \\
\hline 84 & I 6 & 3.2 \\
\hline 85 & » $\quad$ I 9 & 21.6 \\
\hline 86 & "23 & 16.0 \\
\hline 87 & $\gg 27$ & 10.4 \\
\hline 88 & Dez. I & 4.7 \\
\hline 89 & $»$ & 23.1 \\
\hline
\end{tabular}

\begin{tabular}{|c|c|c|c|}
\hline Epoche & $\cdot \quad 19 \mathrm{I}$ & & M.Z.Gr. \\
\hline 2490 & Dez. & 8 & I $7^{\mathrm{h}} \cdot 5$ \\
\hline $9 \mathrm{I}$ & $»$ & I 2 & I I I .9 \\
\hline 92 & $»$ & I 6 & 6.2 \\
\hline 93 & $\gg$ & 20 & 0.6 \\
\hline 94 & $》$ & 23 & I 9.0 \\
\hline 95 & 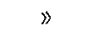 & 27 & I $3 \cdot 4$ \\
\hline 96 & $»$ & $3^{1}$ & 7.8 \\
\hline
\end{tabular}

\title{
Posizione media al 1911.0 della Nova (137.1910) Lacertae di Espin
}

da osservazioni differenziali eseguite nel R. Osservatorio di Capodimonte.

All'epoca nella quale ho potuto osservare questa stella, qualche mese dopo la scoperta, le osservazioni al circolo meridiano non erano possibili, e mi sono perciò avvalso dell'equatoriale di Fraunhofer, di apertura $175 \mathrm{~mm}$, distanza focale $3.02 \mathrm{~m}$, ingrandimento $\mathrm{x} 40$. Il micrometro aveva due terne di fili in ascensione retta, un filo fisso ed uno mobile in declinazione e la vite micrometrico il passo di 22.47 .

Le stelle di paragone furono cinque, tutte a nord della Nova, di grandezze poco diverse tra loro, da $7^{\mathrm{m}} \cdot 6$ a $9^{\mathrm{m}} \mathrm{I}$, e prossime a quella della Nova, da me stimata di $9^{\mathrm{m}} \cdot 2$ durante le osservazioni. Le posizioni medie delle stelle ricavate da quelle del catalogo sono le seguenti:

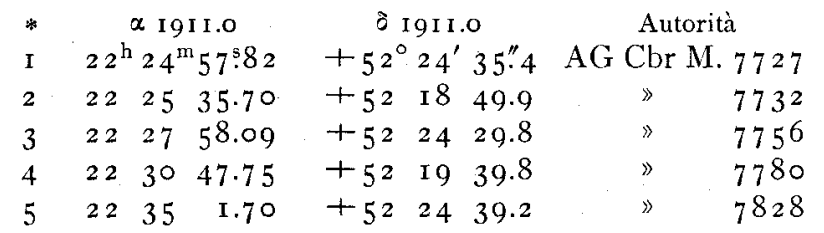

I paragoni sono stati eseguiti dal $2 \mathbf{1}$ Febbraio al I 9 Marzo, durante I 3 serate, quasi sempre facendo la puntata in declinazione in mezzo alle due terne di appulsi in ascensione retta, ed osservando in ogni paragone tutte le stelle, meno alcune volte la $* 2$, perchè traversava i primi fili mentre la * I non ancora era uscita dagli ultimi.

Ciascuna differenza os servata $\left(\alpha_{N}-\alpha *\right)_{0}$ è stata corretta dall'errore di orientamento $\Delta \omega$ del micrometro, compresovi l'effetto della refrazione, è stata ancora ridotta al principio dell'anno e poi sommata con $\alpha * ; \omega$ è risultato, col metodo dei minimi quadrati, dalle differenze tra le ascensioni rette calcolate ed osservate delle $* 1, * 3, * 5$, le quali stanno quasi sopra uno stesso parallelo, combinate con la $* 2$ e con la $* 4$.

Ciascuna differenza osservata $\left(\delta_{N}-\delta_{*}\right)_{0}$ è stata corretta dall'errore dovuto alla refrazione, è stata ridotta al principio dell'anno, e sommata con $\delta_{*}$. Ho trascurato l'errore di orientamento perchè minore di o." $\mathrm{I}$.

Qui appresso riporto $\mathrm{i}$ valori medii delle coordinate $\alpha$ e $\delta$ della Nova, ottenuti da ciascuna stella, seguiti dal numero $n$ dei paragoni, dall'errore probabile $\varepsilon_{m}$ e dal peso relativo $p$ che corrisponde a quest'ultimo; tenendo conto che per le ascensioni rette ciascun valore di $n$ si deve considerare come medio dei passaggi a tutti i sei fili orarii del reticolo.

\begin{tabular}{|c|c|c|c|c|c|c|c|c|}
\hline * & ce I9II.O & $n$ & $\varepsilon_{m}$ & $p$ & $\delta$ I 9 II.० & $n$ & $\varepsilon_{m}$ & $p$ \\
\hline I & $22^{\mathrm{h}} 32^{\mathrm{m}}$ I I .75 & 23 & \pm 0.025 & I 48 & $+52^{\circ} \times 5^{\prime} 20^{\prime \prime} .4$ & 26 & \pm 0.17 & 298 \\
\hline 2 & I 1.64 & 9 & \pm 0.032 & 90 & $2 \mathrm{I} .4$ & 9 & \pm 0.25 & 90 \\
\hline 3 & I 1.86 & 23 & \pm 0.043 & 50 & 19.9 & 29 & \pm 0.16 & 220 \\
\hline 4 & I I. 74 & 28 & \pm 0.024 & 160 & 20.4 & 28 & \pm 0.18 & I 56 \\
\hline 5 & I 1.76 & 23 & \pm 0.029 & I IO & 20.3 & 29 & \pm 0.15 & 250 \\
\hline Med. & $22 \quad 32 \quad 1 \quad 1.74$ & & & & $+5^{2} 1520.4$ & & & \\
\hline
\end{tabular}

Napoli, R. Osservatorio Astronomico di Capodimonte, 3 I Maggio I9 I I.

Eugenio Guerrieri.

\section{Zur bevorstehenden Opposition des Mars.}

Gegen meine aus dem Jahre I908 stammende Erklärung (vergl. A. N. 4348, 4398) des Mars ist mir bisher keine wesentliche Einwendung bekannt geworden. Es sind im Gegenteil immer noch günstige Anzeichen zu den bisher veröffentlichten hinzugekommen. Ich gestatte mir daher, die Herren Beobachter einzuladen, bei der diesjährigen Opposition des Mars die Erscheinungen zur Beurteilung meiner Hypothese zu benutzen.
Ein Hinweis auf die besonders zu beachtenden Orte ist zwar sehr erwünscht, aber vom Standpunkt meiner Erklärung gewagt, weil diese eine ungeheure und selbstredend unberechenbare vulkanische Tätigkeit auf dem Mars voraussetzt. Wenn dieselbe sich jetzt nicht besonders bemerkbar macht, dürften die folgenden Merkmale hervortreten. Der Lacus Solis dürfte wieder etwas kleiner und die benachbarte Thaumasia etwas heller erscheinen, weil der auf das Eis 